\title{
Capsule endoscopy findings for the diagnosis of Crohn's disease: a nationwide case-control study
}

\author{
Motohiro Esaki $^{1}$ (D) Takayuki Matsumoto $^{2}$ - Naoki Ohmiya ${ }^{3} \cdot$ Ema Washio $^{1}$ • \\ Toshifumi Morishita $^{1} \cdot$ Kei Sakamoto $^{1} \cdot$ Hiroo Abe $^{4} \cdot$ Shojiro Yamamoto $^{4}$. \\ Tetsu Kinjo $^{5}$ - Kazutomo Togashi ${ }^{6} \cdot$ Kenji Watanabe $^{7}$. Fumihito Hirai ${ }^{8}$. \\ Masanao Nakamura ${ }^{9}$ Sadaharu Nouda ${ }^{10} \cdot$ Shinya Ashizuka ${ }^{11} \cdot$ Teppei Omori $^{12}$. \\ Shuji Kochi ${ }^{13}$ - Shunichi Yanai ${ }^{2} \cdot$ Yuta Fuyuno $^{1} \cdot$ Atsushi Hirano $^{1}$. \\ Junji Umeno $^{1}$ - Takanari Kitazono ${ }^{1} \cdot$ Fukunori Kinjo $^{14} \cdot$ Mamoru Watanabe $^{15}$. \\ Toshiyuki Matsui $^{8} \cdot$ Yasuo Suzuki $^{16}$
}

Received: 10 April 2018/Accepted: 4 September 2018/Published online: 15 September 2018

(C) The Author(s) 2018

\begin{abstract}
Background Capsule endoscopy can be used to identify the early stage of small bowel Crohn's disease (CD). We evaluated significant small bowel capsule endoscopy (SBCE) findings that can lead to early diagnosis of CD. Methods We retrospectively accumulated clinical and SBCE data of 108 patients (63 with and 45 without CD). Types of small bowel mucosal injuries, including erosion,
\end{abstract}

Electronic supplementary material The online version of this article (https://doi.org/10.1007/s00535-018-1507-6) contains supplementary material, which is available to authorized users.

Motohiro Esaki

mesaki@intmed2.med.kyushu-u.ac.jp

1 Department of Medicine and Clinical Science, Graduate School of Medical Sciences, Kyushu University, Maidashi 3-1-1, Higashi-ku, Fukuoka 812-8582, Japan

2 Division of Gastroenterology, Department of Internal Medicine, School of Medicine, Iwate Medical University, Morioka, Japan

3 Department of Gastroenterology, Fujita Health University School of Medicine, Toyoake, Japan

4 Department of Gastroenterology and Hematology, Faculty of Medicine, University of Miyazaki, Miyazaki, Japan

5 Department of Endoscopy, University of Ryukyus Hospital, Nishihara, Okinawa, Japan

6 Department of Coloproctology, Aizu Medical Center, Fukushima Medical University, Aizuwakamatsu-City, Japan

7 Department of Intestinal Inflammation Research, Hyogo College of Medicine, Nishinomiya, Hyogo, Japan

8 Inflammatory Bowel Disease Center, Fukuoka University Chikushi Hospital, Chikushino, Japan ulceration, and cobblestone appearance, and the alignment of diminutive lesions were compared between patients with and without $\mathrm{CD}$. Inter- and intra-observer agreement in the determination of lesions was assessed in 25 pairs of SBCE from the two groups.

Results Under SBCE, cobblestone appearance (33\% vs. $2 \%, p<0.0001)$, longitudinal ulcers $(78 \%$ vs. $20 \%$, $p<0.0001)$, and irregular ulcers $(84 \%$ vs. $60 \%, p<0.01)$ were more frequently found in patients with $\mathrm{CD}$. Linear erosion $(90 \%$ vs. $38 \%, p<0.0001)$ and irregular erosion $(89 \%$ vs. $64 \%, p<0.005)$ were also more frequent in

9 Department of Gastroenterology and Hepatology, Nagoya University Graduate School of Medicine, Nagoya, Japan

10 Second Department of Internal Medicine, Osaka Medical College, Takatsuki, Japan

11 Department of Circulatory and Body Fluid Regulation, Faculty of Medicine, University of Miyazaki, Miyazaki, Japan

12 Institute of Gastroenterology, Tokyo Women's Medical University, Tokyo, Japan

13 Division of Gastroenterology, Matsuyama Red Cross Hospital, Matsuyama, Japan

14 Center for Gastroenterology, Urasoe General Hospital, Urasoe, Japan

15 Department of Gastroenterology, School of Medicine, Tokyo Medical and Dental University, Tokyo, Japan

16 Department of Internal Medicine, Toho University, Sakura Medical Centre, Sakura, Chiba, Japan 
patients with CD. Furthermore, circumferential (75\% vs. $9 \%, p<0.0001)$ and longitudinal $(56 \%$ vs. $7 \%$, $p<0.0001$ ) alignment of diminutive lesions, mainly observed in the 1st tertile of the small bowel, was more frequent in patients with $\mathrm{CD}$. Good intra-observer agreement was found for ulcers, cobblestone appearance, and lesion alignment. However, inter-observer agreement of SBCE findings differed among observers.

Conclusions Circumferential or longitudinal alignment of diminutive lesions, especially in the upper small bowel, may be a diagnostic clue for CD under SBCE, while interobserver variations should be cautiously considered when using SBCE.

Keywords Capsule endoscopy · Crohn's disease . Diagnostic accuracy

$\begin{array}{ll}\text { Abbreviations } \\ \text { SBCE } & \text { Small bowel capsule endoscopy } \\ \text { CD } & \text { Crohn's disease } \\ \text { IBD } & \text { Inflammatory bowel diseases } \\ \text { NSAIDs } & \text { Nonsteroidal anti-inflammatory drug }\end{array}$

\section{Introduction}

As evidence has accumulated regarding the use of small bowel capsule endoscopy (SBCE), this procedure has been considered useful for the diagnosis of small bowel pathologies [1-4]. SBCE is invaluable for identification of small bowel lesions, mucosal healing, and postoperative recurrence in patients with suspected or established diagnosis of Crohn's disease (CD) [5-10].

While SBCE is not a standard technique for the diagnosis of $\mathrm{CD}$, several reports have suggested favorable diagnostic yields of the procedure in patients with suspected CD [11-15]. A meta-analysis also suggested the superiority of SBCE for the diagnosis of $\mathrm{CD}$ in comparison with small bowel radiography, push enteroscopy, and magnetic resonance enterography [16]. However, the criteria for SBCE-based diagnosis of CD vary widely among previous clinical trials.

Mow et al. [17] suggested that $>3$ ulcerations under SBCE and a negative history for the use of nonsteroidal anti-inflammatory drugs (NSAIDs) are diagnostic for CD in patients suspected to have this disease. However, considering the characteristic mucosal lesions in patients with $\mathrm{CD}$, such as longitudinal ulcers and cobblestone appearance [18-21], the types and predominant location of mucosal lesions need to be seriously considered when establishing SBCE-based diagnosis of CD. Furthermore, diminutive mucosal lesions such as aphthous lesions or erosions have been considered the precursor lesions of $\mathrm{CD}$ $[18,19,22]$. In particular, the lesions aligned longitudinally develop into longitudinal ulcers with the progress of the disease [22]. However, no other characteristic findings are determined to date that help discriminate the early stage of $\mathrm{CD}$ from other miscellaneous diseases. In this sense, SBCE can be useful because it is superior to other modalities for the depiction of diminutive mucosal lesions of the small bowel.

In the present study, we aimed to identify the SBCE findings that lead to the diagnosis of the early stage of $\mathrm{CD}$. Furthermore, inter-observer and intra-observer variations in SBCE findings were investigated to ascertain the clinical usefulness of SBCE.

\section{Materials and methods}

\section{Clinical and SBCE data accumulation}

The present study was based on a collection of nationwide SBCE data from institutions specializing in inflammatory bowel diseases (IBD). The inclusion criteria were (1) a negative history of recent NSAIDs use (within 2 months), (2) a suspicion of having IBD, and (3) positive small bowel mucosal injuries under total enteroscopy by SBCE at the initial diagnosis. We retrospectively accumulated clinical and SBCE data of 116 patients either with or without a final diagnosis of $\mathrm{CD}$. However, eight patients were excluded after reviewing SBCE data (scarred small bowel ulcers alone in four, failure of total enteroscopy in two, and absence of small bowel mucosal lesions in two). Consequently, a total of 108 patients were the subjects of the present study.

The clinical data of each patient were obtained using a query sheet containing five queries: (1) age at the time of SBCE and sex, (2) final diagnosis, (3) indication for SBCE, (4) lesions in the gastrointestinal tract as determined by other imaging modalities at the initial diagnosis, and (5) laboratory data at the time of SBCE including the white blood cell count, hemoglobin level, platelet count, serum total protein, albumin level, and C-reactive protein level. The final diagnosis was subsequently determined based on clinical symptoms, laboratory data, and findings observed by conventional imaging modalities at each participating institution. The diagnosis of CD was based on the Japanese diagnostic criteria for CD (Supplementary Table S1) [19].

All the query sheet and full set of SBCE images were sent to our institution (Department of Medicine and Clinical Sciences, Kyushu University) and stored until analysis. The study protocol was approved by the ethics committee at Kyushu University Hospital (approved No. 24-135) and 
other participating institutions, and the study was conducted in accordance with the Helsinki Declaration.

\section{SBCE assessment}

All digital video image streams of SBCE (PillCam SB2 and SB3; Given Imaging, Ltd., Yokneam, Israel) were downloaded with anonymization to the Given Imaging Reporting and Processing of Images and Data (RAPID) system. An expert capsule endoscopist (M.E.) who was blinded to the clinical information of each patient reviewed the SBCE images under the following reading protocol. SBCE images were carefully analyzed at a maximum rate of 14 frames per s with concurrent manual viewing for close assessment under the dual mode of the Multiview system.
SBCE video images were divided into three tertiles according to the small bowel transit time. Small bowel mucosal injuries were classified as erosions, ulcers, and cobblestone appearance (Fig. 1a-c). The types of erosions or ulcers were further classified as oval, irregular, linear/longitudinal, or circular (Fig. 2a-d). An erosion was defined as mucosal break of $<3 \mathrm{~mm}$ under SBCE, while a larger mucosal defect was defined as an ulcer [23, 24]. As for linear/longitudinal or circular mucosal injuries, the classification between erosion and ulcer was based on the minor axis $(<3 \mathrm{~mm})$ of the mucosal defects. Among small bowel mucosal injuries, erosions and small ulcers of any types were defined as diminutive lesions. When multiple erosions or ulcers were observed in a patient, we determined the types of each mucosal lesion. The alignment of
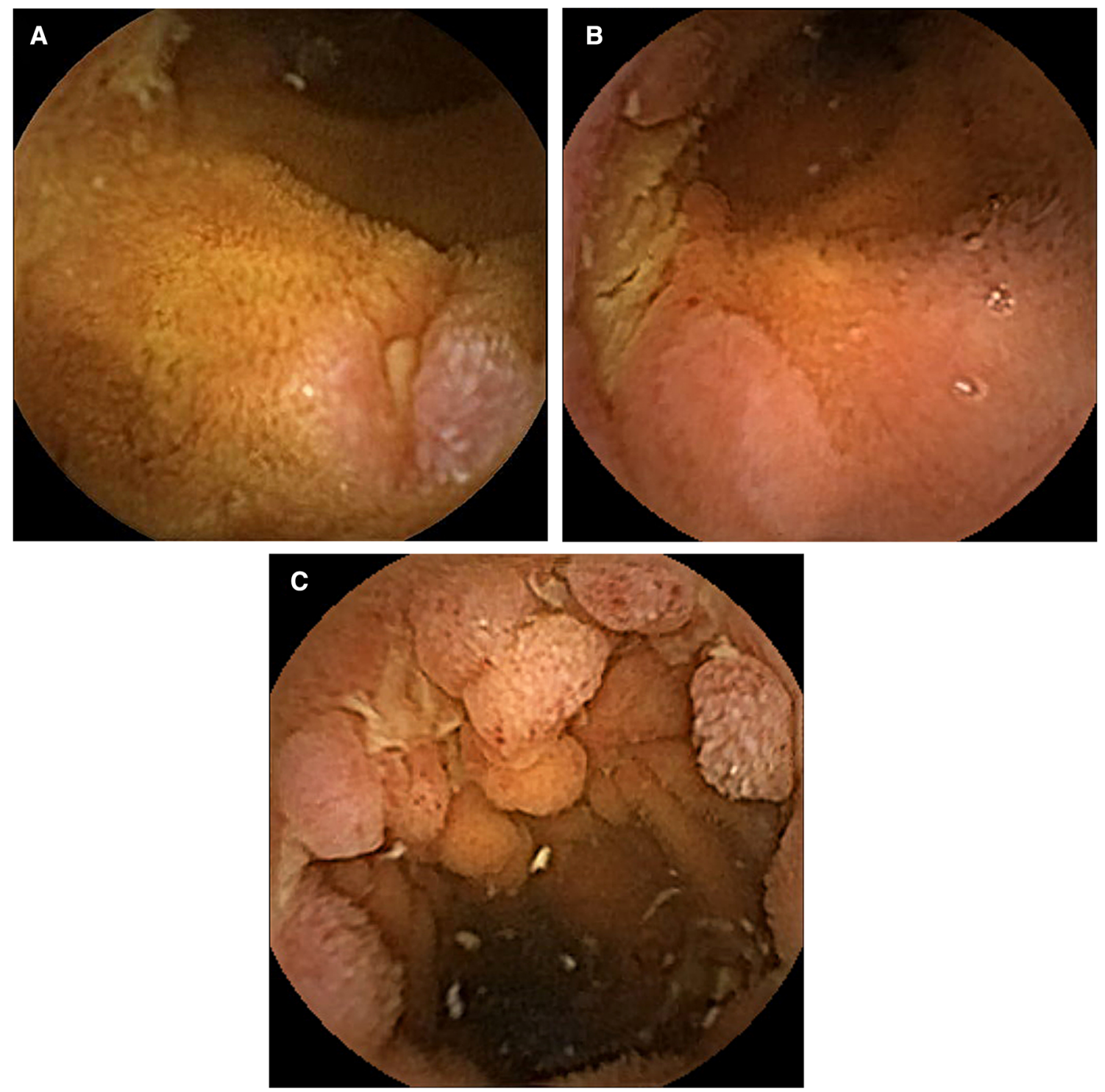

Fig. 1 Classification of mucosal lesions under SBCE. SBCE demonstrates an a erosion, b ulcer, and c cobblestone appearance of the small bowel 

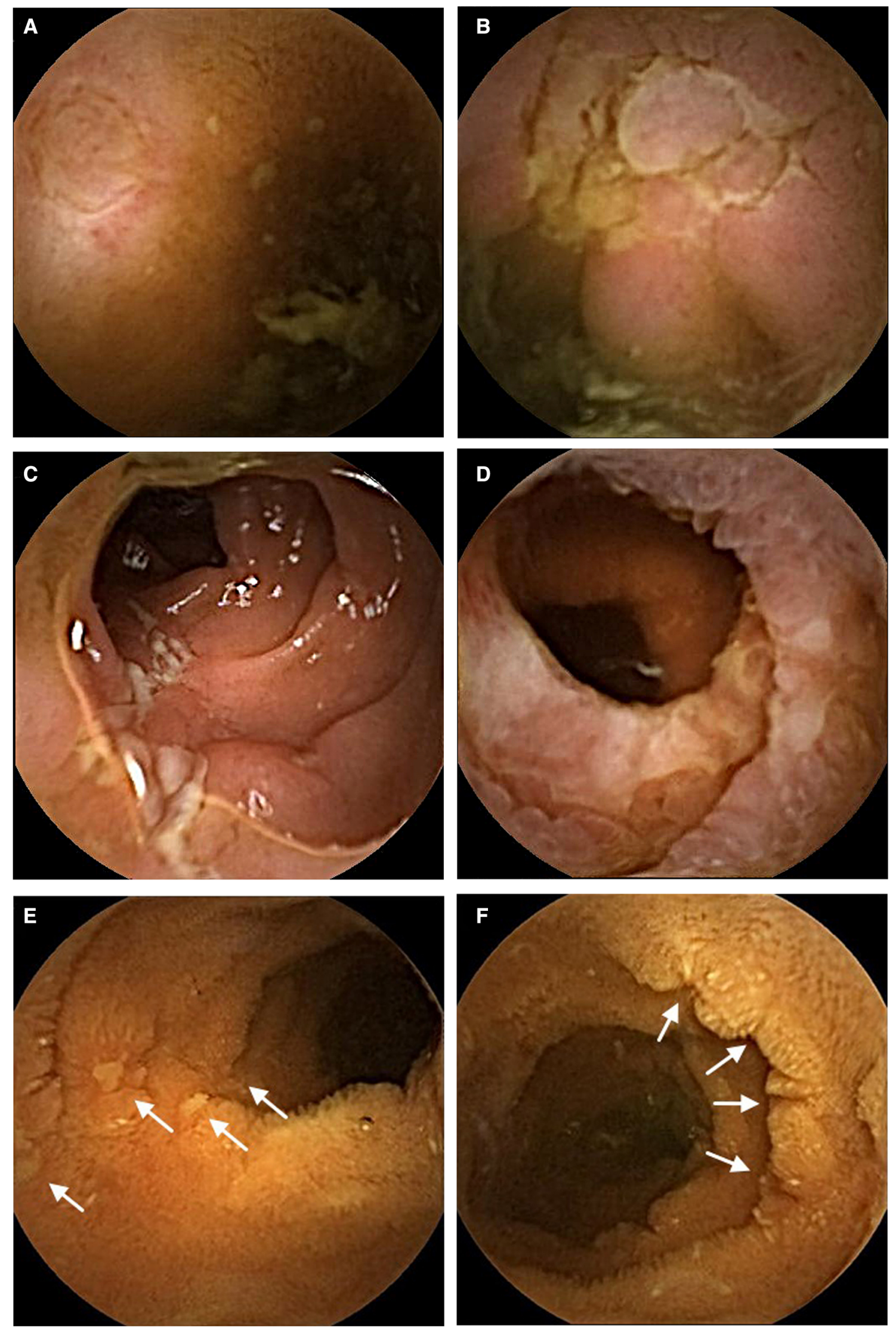
4Fig. 2 Classification of types of mucosal lesions and lesion alignment. SBCE demonstrates an a oval, b irregular, c longitudinal, and d circular ulcer. SBCE depicts, e longitudinal or $\mathbf{f}$ circumferential alignment of diminutive lesions (arrows)

three or more diminutive lesions was classified as longitudinal or circumferential (Fig. 2e, f).

Finally, the overall score for small bowel mucosal injuries was calculated in accordance with the formula for the Lewis score [25]. In each tertile, small bowel cleanliness was assessed and graded on a 4-point scale according to the definition established by Brotz et al. [26].

\section{Assessment of inter- and intra-observer agreement}

Among the 108 patients with SBCE data, we selected 25 patients with $\mathrm{CD}$ and 25 patients without $\mathrm{CD}$ for inter- and intra-observer variation analyses. The severity of small bowel mucosal injuries and the small bowel cleanliness was generally matched in each pair of SBCE. Three observers (E.M., T.M., and K.S.), who had different levels of clinical and SBCE experience, independently reviewed the SBCE data for inter-observer variation analyses. One observer (K.S.) had 2 years of clinical experience as a gastroenterologist and had read ten SBCE videos, another (T.M.) had 8 years of clinical experience as a gastroenterologist and had read 30 SBCE videos, and the remaining observer (E.M.) had 8 years of clinical experience and had read more than 200 SBCE videos. All observers were asked to review all $50 \mathrm{SBCE}$ videos within 3 months following the specific reading protocol. The established protocol specified the order of SBCE videos, the maximum frame rate (14 frames per s) with concurrent manual viewing, and the modality of reading videos (dual mode). Each observer was instructed regarding the reporting protocol using representative images before viewing SBCE data, and the SBCE findings were recorded in predefined formats. For the intra-observer variation analysis, one observer (M.E.) repeatedly evaluated the identical SBCE records at an interval of more than 1 year under the same reading protocol.

All four observers were completely blinded to the patients' final diagnosis. The results of the first SBCE analysis by an expert were used as the reference standard for the inter- and intra-observer variation analyses.

\section{Statistical analysis}

Categorical data are expressed as frequencies and percentages, and numerical variables are expressed as mean \pm standard deviation or median [interquartile range]. Clinical characteristics and SBCE findings were compared between patients with and without $\mathrm{CD}$ using the Chi squared test, Fisher's exact probability test, or the MannWhitney $U$ test, where appropriate. The Wilcoxon signedrank test or McNemar test was used to compare SBCE findings according to the small bowel segment in patients with CD. Spearman's rank sum test was used to calculate the correlations between the Lewis score and hematological parameters. The kappa ( $\kappa$ ) statistic [27] was used to measure the agreement between each observer and the reference standard. The $\kappa$ includes a correction for the amount of agreement that would be expected by chance alone. The $\kappa$ index ranges from 0 (absence of agreement) to 1 (perfect agreement). A value of $<0.20$ indicates slight agreement, from 0.21 to 0.40 fair agreement, from 0.41 to 0.60 moderate agreement, from 0.61 to 0.80 good agreement, and $>0.81$ excellent agreement [28]. The results are reported as the mean $\kappa$ with $95 \%$ confidence interval. The analyses were performed using JMP Pro statistical package 12.2.0 (SAS Institute, Cary, NC, USA). A $p$ value of $<0.05$ was considered statistically significant, and Bonferroni correction was used in the multiple comparison procedure.

\section{Results}

\section{Patients}

Supplementary Table S2 shows the diagnoses of the 108 patients. In addition to SBCE, ileocolonoscopy was performed in 79, esophagogastroduodenoscopy in 48, abdominal CT in 27 , small bowel radiography in 21 , retrograde balloon-assisted enteroscopy in 10, and abdominal US in five patients for the diagnoses. The study cohort comprised 63 patients with $\mathrm{CD}$ and 45 patients without $\mathrm{CD}$. Final diagnoses were established in 32 patients without $\mathrm{CD}$, while the diagnosis remained obscure or was IBDunclassified in the remaining 13 patients. Patients with $C D$ were younger and more frequently had perianal lesions than patients without $\mathrm{CD}$. The serum total protein level was lower in patients without $\mathrm{CD}$, whereas the serum albumin level was not different between the two groups (Supplementary Table S3).

\section{Comparison of SBCE findings between patients with and without $\mathrm{CD}$}

Table 1 compares the SBCE findings between patients with and without $\mathrm{CD}$. With respect to major lesions including ulcers and cobblestone appearance, the number of ulcers was greater in patients with than without CD (9 [2-20] vs. 2 [0-7], $p=0.0001)$, and irregular ulcers $(84 \%$ vs. $60 \%$, $p=0.007)$ and longitudinal ulcers $(78 \%$ vs. $20 \%$, 
Table 1 Comparison of SBCE findings between patients with and without $\mathrm{CD}$

\begin{tabular}{|c|c|c|c|}
\hline & CD group & Non-CD group & $p$ value \\
\hline Number of ulcer & $9[2-20]$ & $2[0-7]$ & 0.0001 \\
\hline \multicolumn{4}{|l|}{ Types of ulcer } \\
\hline Oval & $18(29 \%)$ & $14(31 \%)$ & 0.83 \\
\hline Irregular & $53(84 \%)$ & $27(60 \%)$ & 0.0071 \\
\hline Longitudinal & $49(78 \%)$ & $9(20 \%)$ & $<0.0001$ \\
\hline Circular & $12(19 \%)$ & $8(18 \%)$ & 1.0 \\
\hline Cobblestone appearance & $21(33 \%)$ & $1(2 \%)$ & $<0.0001$ \\
\hline Number of erosion & $42[18-93]$ & $8[4-24.5]$ & $<0.0001$ \\
\hline \multicolumn{4}{|l|}{ Types of erosion } \\
\hline Oval & $47(75 \%)$ & $32(71 \%)$ & 0.83 \\
\hline Irregular & $56(89 \%)$ & $29(64 \%)$ & 0.0037 \\
\hline Linear & $57(90 \%)$ & $17(38 \%)$ & $<0.0001$ \\
\hline Circular & $21(33 \%)$ & $9(20 \%)$ & 0.19 \\
\hline \multicolumn{4}{|c|}{ Alignment of diminutive lesions } \\
\hline Longitudinal & $35(56 \%)$ & $3(7 \%)$ & $<0.0001$ \\
\hline Circumferential & $47(75 \%)$ & $4(9 \%)$ & $<0.0001$ \\
\hline Lewis score & 450 [225-1012] & 225 [68-525] & 0.0055 \\
\hline Bowel cleanliness score & $9[8-10]$ & 9 [7-10.5] & 0.77 \\
\hline
\end{tabular}

$p<0.0001)$ were more frequent in patients with than without CD. Cobblestone appearance was found more frequently in patients with $\mathrm{CD}(33 \%$ vs. $2 \%, p<0.0001)$. The Lewis score was higher in patients with than without CD (450 [225-1012] vs. 225 [68-525], $p=0.0055)$. The number of erosions was greater in patients with than without CD (42 [18-93] vs. 8 [4-25], $p<0.0001)$, and irregular $(89 \%$ vs. $64 \%, p=0.0037)$ and linear erosions (90\% vs. $38 \%, p<0.0001)$ were more frequent in patients with $\mathrm{CD}$. With respect to the alignment of diminutive lesions, both longitudinal alignment $(56 \%$ vs. $7 \%$, $p<0.0001)$ and circumferential alignment (75\% vs. $9 \%$, $p<0.0001)$ were more frequent in patients with than without CD.

The sum of the bowel cleanliness score of the three tertiles was not different between the two groups (9 [8-10] vs. 9 [7-10.5], $p=0.77)$.

\section{Comparison of SBCE findings among small bowel segments in patients with $\mathrm{CD}$}

Table 2 compares the SBCE findings among the three tertiles of the small bowel in patients with CD (Table 2). The positive rate of ulcers was significantly higher in the 3rd tertile than in the 1 st and the 2 nd tertiles $(p<0.0001$ and $p=0.0001$, respectively). When the rate of each type of ulcer was compared, irregular ulcers (1st tertile vs. 3rd tertile, $p=0.0001$ and 2 nd tertile vs. 3rd tertile, $p=0.0011)$ and longitudinal ulcers (1st tertile vs. 3rd tertile, $p<0.0001$ and 2 nd tertile vs. 3rd tertile, $p=0.0001$ ) were more frequent in the 3 rd tertile of the small bowel. However, the rate of cobblestone appearance was not different among the three tertiles. The rate of erosions was high in all three tertiles of the small bowel, regardless of the type. When compared the positive rate of regular alignment of diminutive lesions, circumferential (1st tertile vs. 3rd tertile, $p=0.0005$, and 2 nd tertile vs. 3rd tertile, $p=0.0043$ ) and longitudinal (1st tertile vs. 3rd tertile, $p=0.0017$, and 2 nd tertile vs. 3rd tertile, $p=0.049$ ) alignment were more frequent in the 1 st tertile of the small bowel.

In terms of bowel cleansing, the bowel cleanliness score was significantly higher in the 1st tertile than in the 2nd and 3rd tertiles $(4[3,4]$ in the 1 st tertile, $3[3,4]$ in the 2 nd tertile, and $2[2,3]$ in the 3 rd tertile, respectively).

\section{Correlation between laboratory data and SBCE findings in patients with $\mathrm{CD}$}

As shown in Table 3, there was a moderate inverse correlation between the serum albumin level and the Lewis score in patients with $\mathrm{CD}$. This correlation became more significant in patients with $\mathrm{CD}$ of isolated small bowel disease. However, no significant correlation was found between the other laboratory data and the Lewis score.

\section{Inter- and intra-observer variations}

The final diagnosis and the comparison of clinical characteristics of the selected 25 pairs of patients with SBCE data are shown in Supplementary Tables S4 and S5. The Lewis score and sum of bowel cleanliness score were not 
Table 2 Comparison of SBCE findings among three tertiles of the small bowel in patients with $\mathrm{CD}$

\begin{tabular}{llll}
\hline & 1 st tertile & 2nd tertile & 3rd tertile \\
\hline $\begin{array}{l}\text { Presence of any ulcers } \\
\text { Types of ulcer }\end{array}$ & $29(46 \%)$ & $32(51 \%)$ & $51(81 \%)$ \\
Oval & $6(10 \%)$ & $7(11 \%)$ & $15(24 \%)$ \\
Irregular & $24(38 \%)$ & $30(48 \%)$ & $46(73 \%)$ \\
Longitudinal & $16(25 \%)$ & $25(40 \%)$ & $44(70 \%)$ \\
Circular & $3(5 \%)$ & $5(8 \%)$ & $7(11 \%)$ \\
Cobblestone appearance & $8(13 \%)$ & $12(19 \%)$ & $11(17 \%)$ \\
Presence of any erosions & $52(83 \%)$ & $58(92 \%)$ & $61(97 \%)$ \\
Types of erosion & & & $32(51 \%)$ \\
Oval & $29(46 \%)$ & $35(56 \%)$ & $47(75 \%)$ \\
Irregular & $36(57 \%)$ & $38(60 \%)$ & $46(73 \%)$ \\
Linear & $38(60 \%)$ & $39(62 \%)$ & $10(16 \%)$ \\
Circular & $7(11 \%)$ & $10(16 \%)$ & $9(14 \%)$ \\
Alignment of diminutive lesions & & $18(29 \%)$ & $10(16 \%)$ \\
Longitudinal & $25(40 \%)$ & $24(38 \%)$ & $2[2,3]$ \\
Circumferential & $32(51 \%)$ & $3[3,4]$ & \\
Bowel cleanliness score & $4[3,4]$ & &
\end{tabular}

Table 3 Correlation of hematological parameters and the Lewis score in patients with $\mathrm{CD}$

\begin{tabular}{lcc}
\hline Hematological parameter & \multicolumn{2}{c}{ Correlation coefficient $(\rho)$} \\
\cline { 2 - 3 } & All $(n=63)$ & Ileitis only $(n=24)$ \\
\hline White blood cell & -0.0174 & -0.0030 \\
Hemoglobin & -0.3274 & -0.3763 \\
Platelet & 0.30110 & 0.2628 \\
Total protein & -0.0340 & -0.1212 \\
Albumin & -0.4814 & -0.5343 \\
C-reactive protein & 0.3566 & 0.2428 \\
\hline
\end{tabular}

different between the patients with and without CD selected for the validation study.

The inter-observer agreement between the observers and the reference standard is summarized in Table 4. The $\kappa$ value for irregular ulcers ranged from 0.35 to 0.62 , showing good agreement between the two observers with greater clinical experience. However, the $\kappa$ value for longitudinal ulcers ranged widely from 0.05 to 0.59 , resulting in moderate agreement in the observer with greater clinical and SBCE reading experience. In contrast, the $\kappa$ value for cobblestone appearance was similar, showing moderate agreement among all three observers. With respect to the alignment of diminutive lesions, the $\kappa$ values for longitudinal and circumferential alignments improved with greater clinical and SBCE reading experience. However, the $\kappa$ value for diminutive lesions showed poor agreement in all observers.
The results of intra-observer agreement for the expert capsule endoscopist are shown in Table 4. The $\kappa$ values for longitudinal ulcers, circular ulcers, and cobblestone appearance were $0.76,0.63$, and 0.83 , respectively, indicating good or excellent agreement. The $\kappa$ values for longitudinal and circumferential alignment of diminutive lesions were 0.72 and 0.74 , respectively, showing good agreement. However, the $\kappa$ value for erosions showed only moderate agreement.

\section{Diagnostic accuracy of SBCE findings for CD}

The diagnostic accuracy of SBCE findings for CD according to the reference standard is summarized in Table 5. The specificity and positive predictive value (PPV) for longitudinal ulcers or cobblestone appearance were favorable. However, these values for regular alignment of diminutive lesions were much higher. While the sensitivity and negative predictive value (NPV) for linear erosions were favorable, the specificity and PPV remained low. When linear erosions and regular alignment of diminutive lesions were combined, no additive effect was found on the accuracy for the diagnosis of $\mathrm{CD}$.

\section{Discussion}

With the increasing use of SBCE in daily clinical practice, this procedure has been suggested to be useful in the diagnosis and management of a variety of disorders such as obscure gastrointestinal bleeding, polyposis syndromes, 
Table 4 Inter- and intra-observer agreement between the observers and reference standard

\begin{tabular}{|c|c|c|c|c|}
\hline \multirow[t]{2}{*}{ SBCE findings } & \multicolumn{3}{|l|}{ Inter-observer agreement $^{\mathrm{a}}$} & \multirow{2}{*}{$\begin{array}{l}\text { Intra-observer agreement } \\
\text { Expert endoscopist }\end{array}$} \\
\hline & Observer 1 & Observer 2 & Observer 3 & \\
\hline \multicolumn{5}{|l|}{ Ulcer } \\
\hline Oval & $-0.004(-0.22-0.22)$ & $0.51(0.26-0.77)$ & $0.05(-0.23-0.33)$ & $0.32(0.04-0.59)$ \\
\hline Irregular & $0.35(0.15-0.55)$ & $0.64(0.42-0.87)$ & $0.62(0.40-0.84)$ & $0.53(0.27-0.78)$ \\
\hline Longitudinal & $0.05(-0.04-0.14)$ & $0.34(0.09-0.59)$ & $0.59(0.37-0.80)$ & $0.76(0.58-0.94)$ \\
\hline Circular & $0.31(0.03-0.58)$ & $0.43(0.14-0.72)$ & $0.54(0.26-0.82)$ & $0.63(0.38-0.88)$ \\
\hline Cobblestone appearance & $0.50(0.16-0.85)$ & $0.50(0.16-0.85)$ & $0.47(0.21-0.73)$ & $0.83(0.61-1)$ \\
\hline \multicolumn{5}{|l|}{ Erosion } \\
\hline Oval & $0.07(-0.12-0.26)$ & $0.19(-0.11-0.49)$ & $-0.12(-0.34-0.10)$ & $0.42(0.10-0.73)$ \\
\hline Irregular & $0.30(-0.01-0.61)$ & $0.25(-0.05-0.55)$ & $0.38(0.09-0.66)$ & $0.49(0.21-0.78)$ \\
\hline Linear & $0.07(-0.09-0.22)$ & $0.17(-0.03-0.38)$ & $0.38(0.10-0.66)$ & $0.57(0.35-0.80)$ \\
\hline Circular & $0.25(-0.02-0.53)$ & $0.29(0.03-0.56)$ & $0.44(0.17-0.72)$ & $0.49(0.21-0.75)$ \\
\hline \multicolumn{5}{|c|}{ Alignment of diminutive lesions } \\
\hline Longitudinal & $0.14(-0.04-0.31)$ & $0.44(0.19-0.69)$ & $0.57(0.36-0.78)$ & $0.74(0.54-0.93)$ \\
\hline Circumferential & $0.30(0.08-0.53)$ & $0.32(0.07-0.58)$ & $0.45(0.21-0.69)$ & $0.72(0.53-0.91)$ \\
\hline
\end{tabular}

${ }^{a}$ Values are expressed as $\kappa$ value $(95 \% \mathrm{CI})$

Table 5 Diagnostic accuracy of SBCE findings for CD

\begin{tabular}{lllll}
\hline SBCE findings & Sensitivity $(\%)$ & Specificity $(\%)$ & PPV (\%) & NPV (\%) \\
\hline Longitudinal ulcer or cobblestone appearance & 77 & 80 & 84 & 72 \\
Linear erosion & 90 & 62 & 77 & 82 \\
Lesion alignment only & & & & \\
$\quad$ Longitudinal & 56 & 93 & 92 & 60 \\
Circumferential & 75 & 91 & 92 & 72 \\
$\quad$ Longitudinal or circumferential & 79 & 87 & 89 & 75 \\
Linear erosion plus lesion alignment & & & & \\
$\quad$ Longitudinal & 54 & 91 & 89 & 59 \\
Circumferential & 71 & 93 & 94 & 70 \\
Longitudinal or circumferential & 75 & 87 & 89 & 71 \\
\hline
\end{tabular}

$P P V$ Positive predictive value, $N P V$ negative predictive value and IBD [1-4]. CD is considered an appropriate indication for SBCE, and higher diagnostic yields of SBCE in patients with suspected or established CD have been demonstrated when compared with other imaging modalities including small bowel radiography, CT enterography, and push enteroscopy [11-16]. However, as noted by Doherty et al. [29], the significantly higher yields of SBCE than of other imaging modalities may be partly attributable to false positives because of the lack of a precise definition of $\mathrm{CD}$ under SBCE. Actually, the presence of $>3$ ulcers under SBCE as the threshold for suspected CD reportedly yields a PPV of up to $50 \%$ for the diagnosis of CD [30]. We, therefore, investigated endoscopic findings that may lead to better diagnostic accuracy of CD under SBCE.
In the present study, a recent history of NSAIDs use was set to be one of the exclusion criteria following the previous studies. Actually, the most widely used but not validated criteria of SBCE for the diagnosis of CD by Mow et al. [17] are defined as $>3$ ulcerations under SBCE and a negative history of NSAIDs use. Such an exclusion criterion might cause selection bias; however, it seems reasonable to consider the fact that NSAIDs use is usually checked at medical interview when diagnosing CD in daily clinical practice.

We initially compared SBCE findings between patients with and without $C D$ to clarify the characteristic findings of $\mathrm{CD}$. We found that major lesions such as irregular ulcers, longitudinal ulcers, and cobblestone appearance were more frequent in patients with $\mathrm{CD}$. This result seems to be 
reasonable because the latter two findings are defined as the major items of the Japanese diagnostic criteria for CD (Supplementary Table S1) [19]. The present study also indicated that linear or irregular erosions and longitudinal or circumferential alignment of diminutive lesions were more frequent in patients with than without CD. Considering that linear erosions and circumferential alignment of mucosal lesions are not specified in the Japanese diagnostic criteria for CD [19], such findings appear to be noteworthy for the diagnosis of CD under SBCE.

We subsequently investigated the inter-observer and intra-observer agreement of SBCE findings to determine their validity. In the inter-observer variation analysis, three observers with different levels of clinical and SBCE reading experience were selected to ascertain the influence of clinical skills on the detection of SBCE findings. We found that longitudinal ulcers and cobblestone appearance achieved moderate agreement but that irregular or linear erosions had poor agreement. The inter-observer agreement for circumferential or longitudinal alignment improved as clinical experience increased. Combined with the results of the intra-observer variation analyses, regular alignment of diminutive lesions seems to be the most useful finding for the diagnosis of CD under SBCE. Poor inter-observer agreement for each type of erosion may be partly explained by the difference in the interpretation of diminutive lesions among the observers, because the configuration of those lesions can be easily influenced by peristalsis or the amount of intestinal fluid under SBCE.

In addition to the longitudinal alignment, circumferential alignment of the diminutive lesions, especially in the 1st tertile of the small bowel, was more frequent in $\mathrm{CD}$, which has not been considered as characteristic by balloonassisted enteroscopy. Such an inconsistent result might be caused by the following reasons; first, antegrade doubleballoon enteroscopy is rarely performed in CD considering the predominant involvement of the ileum. Second, SBCE images observed under physiological condition could facilitate circumferentially aligned diminutive lesions. However, multiple notching on the duodenal mucosal folds is considered as one of the characteristic findings of the upper GI tract in CD [31], which considerably mimics this type of regular alignment. Therefore, circumferential alignment as well as longitudinal one of the diminutive lesions is considered as the characteristic findings of the upper small bowel including the duodenum in $\mathrm{CD}$.

We also investigated the diagnostic accuracy of $\mathrm{CD}$ based on SBCE findings and found higher specificity and PPVs for circumferential or longitudinal alignment than for longitudinal ulcers or cobblestone appearance. Such superiority of circumferential or longitudinal alignment might also be attributed to the visibility in the mucosal lesions, because circumferential or longitudinal alignment was mainly found in the 1st tertile of the small bowel, where the bowel cleansing level was generally good. Conversely, longitudinal ulcers were mainly found in the 3rd tertile of the small bowel, where the presence of intestinal turbid fluid or residues frequently hampers the detection of mucosal lesions under SBCE. From this viewpoint, it seems reasonable to focus on the regular alignment of diminutive lesions in the 1st tertile of the small bowel under SBCE for the diagnosis of CD.

When we compared mucosal lesions among the three segments of the small bowel in patients with $\mathrm{CD}$, nearly half of the patients had major lesions in the upper twothirds of the small bowel. This result is consistent with previous reports showing a high incidence of jejunal involvement under SBCE $[32,33]$. The present study also demonstrated a considerably high incidence of diminutive lesions throughout the small bowel. Because jejunal involvement has been suggested to be a possible poor prognostic factor for small bowel CD [33], additional studies investigating the clinical impact of jejunal involvement according to the type of lesions seem necessary.

We also analyzed the possible association between hematological parameters and the Lewis score in patients with $\mathrm{CD}$, for whom only a moderate inverse correlation between the serum albumin level and the Lewis score was identified. He et al. [34] recently investigated the relationship of the Lewis score with clinical disease activity indices and the $\mathrm{C}$-reactive protein level in pediatric and adult patients with $\mathrm{CD}$ showed a weak and moderate correlation with the Lewis score, respectively. In contrast, Kopylov et al. [35] performed a prospective study of patients with quiescent or mildly symptomatic small bowel $\mathrm{CD}$ and reported that only a minority of patients with $\mathrm{CD}$ exhibiting clinical and biomarker remission (4.7\%) achieved small bowel mucosal healing. It thus can be assumed that small bowel endoscopic evaluation is crucial for the precise evaluation of small bowel disease activity in patients with $\mathrm{CD}$, while further studies comparing small bowel endoscopic activity with fecal biomarkers such as calprotectin or lactoferrin are required [36-38].

The present study has some limitations. First, it was conducted in a retrospective fashion. However, the observers analyzed anonymized SBCE data and were blinded to the clinical information. We thus believe that the retrospective nature of the study had a minimal effect on our results. Second, the study subjects were limited to the patients having positive small bowel mucosal injuries under SBCE, while the major advantage of the procedure is to exert high NPV for the diagnosis of small bowel CD [1-3]. However, the present study aimed to identify SBCE findings that could facilitate the identification of early CD. Since no such investigation has been reported so far, we 
believe that the present study has an impact when considering the role of SBCE for the diagnosis of CD. Third, the final diagnoses of the enrolled patients were determined by the gastroenterologists at each institution. However, we accumulated SBCE data from institutions specializing in IBD, and the diagnosis of CD is generally made according to the Japanese diagnostic criteria for CD [19]. Therefore, the diagnosis of $\mathrm{CD}$ in our patients is considered to have been reliable. Fourth, the patients without CD included 13 patients with an unconfirmed diagnosis. It is, therefore, possible that patients with $\mathrm{CD}$ who did not meet the diagnostic criteria (e.g., those with a very early stage of CD) might have been included in the group of patients without $\mathrm{CD}$. However, such a misclassification of the very early stage of $\mathrm{CD}$ would have rather strengthened our results. Finally, we could not compare SBCE findings with other imaging modalities including balloon-assisted enteroscopy, which might hamper the reliability of our results. Prospective comparative studies are necessary, while the determination of characteristic SBCE findings for the diagnosis of $\mathrm{CD}$ seems also important.

In conclusion, circumferential or longitudinal alignment of diminutive lesions, especially in the upper small bowel, under SBCE can be a clue for the diagnosis of $\mathrm{CD}$, while a certain level of clinical and SBCE experience is mandatory for accurate detection. Further prospective studies are necessary to ascertain the clinical usefulness of SBCE and determine its true diagnostic yield in patients with suspected CD.

Acknowledgements The authors are grateful to the following doctors, who significantly contributed to the present work: Takahiro Wakamatsu, MD (Third Department of Internal Medicine, Kansai Medical University, Osaka, Japan); Naoki Hosoe, MD (Center for Diagnostic and Therapeutic Endoscopy, School of Medicine, Keio University, Tokyo, Japan); Jun Kato, MD (Second Department of Internal Medicine, Wakayama Medical University, Wakayama, Japan); Mikihiro Fujiya, MD (Division of Gastroenterology and Hematological Oncology, Department of Medicine, Asahikawa Medical University, Asahikawa, Japan); Takashi Joh, MD (Department of Gastroenterology and Metabolism, Nagoya City University Graduate School of Medical Sciences, Nagoya, Japan); Katsuya Endo, MD (Division of Gastroenterology, Tohoku University Graduate School of Medicine, Sendai, Japan); Hiroki Endo, MD (Department of Gastroenterology and Hepatology, Yokohama City University School of Medicine, Yokohama, Japan); Osamu Handa, MD (Department of Molecular Gastroenterology and Hepatology, Kyoto Prefectural University of Medicine, Graduate School of Medical Science, Kyoto, Japan); Reiko Kunisaki, MD (IBD Center, Yokohama City University Medical Center, Yokohama, Japan); and Makoto Sasaki, MD (Department of Gastroenterology, Division of Internal Medicine, Aichi Medical University School of Medicine, Nagoya, Japan).

Authors' contributions The authors' contributions to the manuscript are as follows: study concept and design (ME, TM, AH, JU), acquisition of data (NO, SY, TK, KT, KW, FH, MN, SN, SA, TO, SK, SY, $\mathrm{YF}, \mathrm{AH})$, analysis and interpretation of data (ME, TM, EW, TM, KS,
JU), drafting of the manuscript (ME, TM, TM), critical revision of the manuscript for important intellectual content (TK, FK, YS), statistical analysis (JU), and study supervision (MW, TM, YS).

Funding This work was supported in part by Health and Labour Sciences Grants for research on intractable diseases from Ministry of Health, Labour and Welfare of Japan (Investigation and Research for Intractable Inflammatory Bowel Disease). The funder of the study had no role in the study design, data collection, data analysis, data interpretation, or writing of the report.

\section{Compliance with ethical standards}

Conflict of interest Motohiro Esaki received research grants from, Mitsubishi Tanabe Pharma Corporation, EA Pharma Co., Ltd., AbbVie GK, ZERIA Pharmaceutical Co., Ltd., and Asahi Kasei Medical Co., Ltd., and lecture fee from Mitsubishi Tanabe Pharma Corporation and AbbVie GK, outside the submitted work. Takayuki Matsumoto received research grants from Mitsubishi Tanabe Pharma Corporation, EA Pharma Co., Ltd., and Nippon Kayaku Co., Ltd., and lecture fee from Mitsubishi Tanabe Pharma Corporation, EA Pharma Co., Ltd., KYORIN Pharmaceutical Co., Ltd., AbbVie GK, Janssen Pharmaceutical K.K., Mochida Pharmaceutical Co., Ltd., Pfizer Inc., Zeria Pharmaceutical Co., Ltd., and AstraZeneca plc, and advisory fee from JIMRO Co., Ltd., Kissei Pharmaceutical Co., Ltd., and Takeda Pharmaceutical Co., Ltd., outside the submitted work. Naoki Ohmiya received research grants from Otsuka Pharamaceutical Factory, Inc., JIMRO Co., Ltd., Mochida Pharmaceutical Co., Ltd., Daiichi Sankyo Company, Limited, Astellas Pharma Inc., Eli Lilly and Company, Mitsubishi Tanabe Pharma Corporation, EA Pharma Co., Ltd., TAIHO Pharmaceutical Co. Ltd., Takeda Pharmaceutical Co., Ltd., Tsumura \& Co., KYORIN Pharmaceutical Co., Ltd., and Asahi kasei Pharma, and lecture fee from KYORIN Pharmaceutical Co., Ltd., Fujifilm medical Co., Covidien Japan, EA Pharma Co. Ltd., KYORIN Pharmaceutical Co., Ltd., Mitsubishi Tanabe Pharma Corporation, Takeda Pharmaceutical Co., Ltd., ZERIA Pharmaceutical Co., Ltd., Astellas Pharma Inc., AbbVie GK, and Mylan Inc, outside the submitted work. Kenji Watanabe received research grants from AbbVie GK, Mitsubishi Tanabe Pharma Corporation, EA Pharma Co., Ltd., Asahi Kasei Medical Co., Ltd., Kyowa Hakko Kirin Co., Ltd, Kyorin Pharmaceutical Co., Ltd, Astellas Pharma Inc, and JIMRO Co., Ltd., and consulting fee from EA Pharma Co., Ltd., Mitsubishi Tanabe Pharma Corporation, AbbVie GK, Kyorin Pharmaceutical Co., Ltd., Asahi Kasei Medical Co. Ltd., Kyowa Hakko Kirin Co., Ltd., Takeda Pharmaceutical Co., Ltd., JIMRO Co., Ltd., Olympus Corporation, Covidien Japan Co., Ltd., Mochida Pharmaceutical Co., Ltd., Janssen Pharmaceutical K.K., Pfizer Japan Inc., and Otsuka Pharmaceutical Co., Ltd., and lecture fee from Mitsubishi Tanabe Pharma Corporation, Eisai Co., Ltd, AbbVie GK, Asahi Kasei Medical Co., Ltd., Kyowa Hakko Kirin Co., Ltd., Kyorin Pharmaceutical Co., Ltd., Astellas Pharma Inc., JIMRO Co., Ltd., UCB Japan Co., Ltd., Zeria Pharmaceutical Co., Ltd., Takeda Pharmaceutical Co., Ltd., Olympus Corporation, Covidien Japan Co., Ltd., and Otsuka Pharmaceutical Co., Ltd., and advisory fee from Takeda Pharmaceutical Co., Ltd., Mochida Pharmaceutical Co., Ltd., Janssen Pharmaceutical K.K., Pfizer Japan Inc., EA Pharma Co., Ltd., Mitsubishi Tanabe Pharma Corporation, and JIMRO Co., Ltd., outside the submitted work. Fumihito Hirai received research grants from AbbVie GK, Mitsubishi Tanabe Pharma Corporation, EA Pharma Co., Ltd., JIMRO Co., Ltd., ZERIA Pharmaceutical Co., Ltd., and Eisai Co., Ltd., and lecture fee from AbbVie GK, EA Pharma Co., Ltd., Eisai Co., Ltd. And Mitsubishi Tanabe Pharma Corporation, outside the submitted work. Atsushi Hirano received research grants from AbbVie GK, and Mitsubishi Tanabe Pharma Corporation, outside the submitted work. Takanari Kitazono received research grants from Daiichi Sankyo Co., 
Ltd., Takeda Pharmaceutical Co. Ltd., MSD Co., Ltd. Nippon Boehringer Ingelheim Co., Ltd., Sanofi-Aventis Co., Ltd. Pfizer Inc., Bayer yakuhin, Ltd., and Eisai Co., Ltd., and lecture fee from Daiichi Sankyo Co., Ltd., Bayer yakuhin, Ltd., and Chugai Pharmaceutical Co., Ltd., outside the submitted work. Mamoru Watanabe received research grants from Asahi Kasei Medical Co., Ltd., Ajinomoto Co., Inc., AbbVie GK, EA Pharma Co., Ltd., Eisai Co., Ltd., Kyorin Pharmaceutical Co., Ltd., Mitsubishi Tanabe Pharma Corp., Otsuka Pharmaceutical Co., Ltd., Kyowa Hakko Kirin Co. Ltd., Zeria Pharmaceutical Co., Ltd., JIMRO Co., Ltd., Takeda Pharmaceutical Co., Ltd., Nippon Kayaku Co., Ltd., Mochida Pharmaceutical Co., Ltd., Daiichi Sankyo Co., Ltd., Ono Pharmaceutical Co., Ltd., Astellas Pharma Inc., MSD K.K., Dainippon Sumitomo Dainippon Pharma Co., Ltd., Bristol-Myers, K.K, and Chugai Pharmaceutical Co., Ltd., and lecture fees from Mitsubishi Tanabe Pharma Corp., Eisai Co., Ltd., Kyorin Pharmaceutical Co., Ltd., JIMRO Co., Ltd., Ajinomoto Co., Inc., AbbVie GK, Takeda Pharmaceutical Co., Ltd., Kyowa Hakko Kirin Co. Ltd., Zeria Pharmaceutical Co., Ltd., Asahi Kasei Medical Co., Ltd., EA Pharma Co., Ltd., Astellas Pharma Inc., Mochida Pharmaceutical Co., Ltd., Janssen Pharmaceutical Co., Ltd., Gilead Sciences, Inc., Celgene Corp., outside the submitted work. Toshiyuki Matsui received research grants from AbbVie GK, Mitsubishi Tanabe Pharma Corporation, EA Pharma Co., Ltd., JIMRO Co., Ltd., ZERIA Pharmaceutical Co., Ltd., Eisai Co., Ltd., Astellas Pharma Inc, and Takeda Pharmaceutical Co. Ltd., and lecture fee from AbbVie GK, and EA Pharma Co., outside the submitted work. Yasuo Suzuki received research grants from AbbVie GK, Mitsubishi Tanabe Pharma Corporation, Ltd., EA Pharma Co., Ltd., JIMRO Co., Ltd., Mochida Pharmaceutical Co., Ltd., and Nippon Kayaku Co., Ltd., and lecture fee from Mitsubishi Tanabe Pharma Corporation, AbbVie GK, EA Pharma Co. Ltd., ZERIA Pharmaceutical Co., Ltd., Mochida Pharmaceutical Co., Ltd., and KYORIN Pharmaceutical Co., Ltd., outside the submitted work. All other authors have no competing interests to declare.

Open Access This article is distributed under the terms of the Creative Commons Attribution 4.0 International License (http://crea tivecommons.org/licenses/by/4.0/), which permits unrestricted use, distribution, and reproduction in any medium, provided you give appropriate credit to the original author(s) and the source, provide a link to the Creative Commons license, and indicate if changes were made.

\section{References}

1. Pennazio M, Spada C, Eliakim R, et al. Small-bowel capsule endoscopy and device-assisted enteroscopy for diagnosis and treatment of small-bowel disorders: European Society of Gastrointestinal Endoscopy (ESGE) Clinical Guideline. Endoscopy. 2015;47:352-76.

2. Gomollon F, Dignass A, Annese V, et al. 3rd European evidencebased consensus on the diagnosis and management of Crohn's disease 2016: part 1: diagnosis and medical management. J Crohns Colitis. 2017;11:3-25.

3. Enns RA, Hookey L, Armstrong D, et al. Clinical practice guidelines for the use of video capsule endoscopy. Gastroenterology. 2017;152:497-514.

4. Matsuoka K, Kobayashi T, Ueno F, et al. Evidence-based clinical practice guidelines for inflammatory bowel disease. J Gastroenterol. 2018;53:303-53.

5. Gralnek IM, Cohen SA, Ephrath H, et al. Small bowel capsule endoscopy impacts diagnosis and management of pediatric inflammatory bowel disease: a prospective study. Dig Dis Sci. 2012;57:465-71.
6. Efthymiou A, Viazis N, Mantzaris G, et al. Does clinical response correlate with mucosal healing in patients with Crohn's disease of the small bowel? A prospective, case-series study using wireless capsule endoscopy. Inflamm Bowel Dis. 2008;14:1542-7.

7. Hall B, Holleran G, Chin JL, et al. A prospective 52 week mucosal healing assessment of small bowel Crohn's disease as detected by capsule endoscopy. J Crohns Colitis. 2014;8:1601-9.

8. Bourreille A, Jarry M, D'Halluin PN, et al. Wireless capsule endoscopy versus ileocolonoscopy for the diagnosis of postoperative recurrence of Crohn's disease: a prospective study. Gut. 2006;55:978-83.

9. Biancone L, Calabrese E, Petruzziello C, et al. Wireless capsule endoscopy and small intestine contrast ultrasonography in recurrence of Crohn's disease. Inflamm Bowel Dis. 2007;13:1256-65.

10. Esaki M, Matsumoto T, Watanabe $\mathrm{K}$, et al. Use of capsule endoscopy in patients with Crohn's disease in Japan: a multicenter survey. J Gastroenterol Hepatol. 2014;29:96-101.

11. Marmo R, Rotondano G, Piscopo R, et al. Capsule endoscopy versus enteroclysis in the detection of small-bowel involvement in Crohn's disease: a prospective trial. Clin Gastroenterol Hepatol. 2005;3:772-6.

12. Dubcenco E, Jeejeeghoy KN, Petroniene R, et al. Capsule endoscopy findings in patients with established and suspected small-bowel Crohn's disease: correlation with radiologic, endoscopic, and histological findings. Gastrointest Endosc. 2005;62:538-44.

13. Solem CA, Loftus EV Jr, Fletcher JG, et al. Small-bowel imaging in Crohn's disease: a prospective, blinded, 4-way comparison trial. Gastrointest Endosc. 2008;68:255-66.

14. Eliakim R, Suissa A, Yassin K, et al. Wireless capsule endoscopy compared to barium follow-through and computerized tomography in patients with suspected Crohn's disease-final report. Dig Liver Dis. 2004;36:519-22.

15. Hara AK, Leighton JA, Heigh RI, et al. Crohn's disease of the small bowel: preliminary comparison of CT enterography, capsule endoscopy, small-bowel follow-through and ileoscopy. Radiology. 2006;238:128-34.

16. Dionisio PM, Gurudu SR, Leighton JA, et al. Capsule endoscopy has a significantly higher diagnostic yield in patients with suspected and established small-bowel Crohn's disease: a metaanalysis. Am J Gastroenterol. 2010;105:1240-8.

17. Mow WS, Lo SK, Targan SR, et al. Initial experience with wireless capsule enteroscopy in the diagnosis and management of inflammatory bowel disease. Clin Gastroenterol Hepatol. 2004;2:31-40.

18. Yao T, Matsui T, Hiwatashi N. Crohn's disease in Japan: diagnostic criteria and epidemiology. Dis Colon Rectum. 2000;43:S85-93.

19. Hisabe T, Hirai F, Matsui T, et al. Evaluation of diagnostic criteria for Crohn's disease in Japan. J Gastroenterol. 2014;49:93-9.

20. Saibeni S, Rondonotti E, Iozzelli A, et al. Imaging of the small bowel in Crohn's disease: a review of old and new techniques. World J Gastroenterol. 2007;13:3279-87.

21. Nikolaus S, Schreiber S. Diagnostics of inflammatory bowel disease. Gastroenterology. 2007;133:1670-89.

22. Hirai F, Yano Y, Ohara J, et al. Long-term clinical course of Crohn's disease of aphthous type. Stomach Intest. 2005;40:895-910 (Japanese with English abstract).

23. Maehata Y, Esaki M, Morishita T, et al. Small bowel injury induced by selective cyclooxygenase- 2 inhibitors: a prospective, double-blind, randomized clinical trial comparing celecoxib and meloxicam. J Gastroenterol. 2012;47:387-93.

24. Washio W, EsakiM Maehata Y, et al. Proton pump inhibitors increase incidence of nonsteroidal anti-inflammatory drug- 
induced small bowel injury: a randomized, placebo-controlled trial. Clin Gastroenterol Hepatol. 2016;14:809-15.

25. Gralnek IM, Defranchis R, Seidman E, et al. Development of a capsule endoscopy scoring index for small bowel mucosal inflammatory change. Aliment Pharmacol Ther. 2008;27:146-54.

26. Brotz C, Nandi N, Conn M, et al. A validation study of three grading systems to evaluate small-bowel cleansing for wireless capsule endoscopy: a quantitative index, a qualitative evaluation, and an overall adequacy assessment. Gastrointest Endosc. 2009;69:262-70.

27. Fleiss JL. The measurement of interobserver agreement. In: Fleiss JL, editor. Statistical methods for rates and proportions. New York: John Wiley; 1981. p. 212-36.

28. Landis JR, Koch GG. The measurement of observer agreement for categorical data. Biometrics. 1977;33:159-74.

29. Doherty GA, Moss AC, Cheifetz AS. Capsule endoscopy in suspected Crohn's disease: "yield" does not equal "diagnosis". Am J Gastroenterol. 2010;105:2111.

30. Tukey M, Pleskow D, Legnani P, et al. The utility of capsule endoscopy in patients with suspected Crohn's disease. Am J Gastroenterol. 2009;104:2734-9.

31. Yorioka M, Yao T, Sakurai T, et al. Endoscopic findings in duodenum of Crohn's disease patients. Stomach Intest. 2001;36:1481-7 (Japanese with English abstract).

32. Petruzziello C, Onali S, Calabrese E, et al. Wireless capsule endoscopy and proximal small bowel lesions in Crohn's disease. World J Gastroenterol. 2010;16:3299-304.
33. Flamant $\mathrm{M}$, Trang $\mathrm{C}$, Maillard $\mathrm{O}$, et al. The prevalence and outcome of jejunal lesions visualized by small bowel capsule endoscopy in Crohn's disease. Inflamm Bowel Dis. 2013;19:1390-6.

34. He C, Zhang J, Chen Z, et al. Relationships of capsule endoscopy Lewis score with clinical disease activity indices, C-reactive protein, and small bowel transit time in pediatric and adult patients with small bowel Crohn's disease. Medicine (Baltimore). 2017;96:e7780.

35. Kopylov U, Yablecovitch D, Lahat A, et al. Detection of small bowel mucosal healing and deep remission in patients with known small bowel Crohn's disease using biomarkers, capsule endoscopy, and imaging. Am J Gastroenterol. 2015;110:1316-23.

36. Sipponen T, Haapamäki J, Savilahti E, et al. Fecal calprotectin and S100A12 have low utility in prediction of small bowel Crohn's disease detected by wireless capsule endoscopy. Scand J Gastroenterol. 2012;47:778-84.

37. Höög CM, Bark L, Broström O, et al. Capsule endoscopic findings correlate with fecal calprotectin and C-reactive protein in patients with suspected small-bowel Crohn's disease. Scand J Gastroenterol. 2014;49:1084-90.

38. Shitrit AB, Koslowsky B, Livovsky DM, et al. A prospective study of fecal calprotectin and lactoferrin as predictors of small bowel Crohn's disease in patients undergoing capsule endoscopy. Scand J Gastroenterol. 2017;52:328-33. 